03

\title{
Сверхзвуковое ламинарное обтекание затупленного ребра: двойственность численного решения
}

\author{
(C) Е.В. Колесник, Е.М. Смирнов \\ Санкт-Петербургский политехнический университет Петра Великого, \\ 195251 Санкт-Петербург, Россия \\ e-mail: kolesnik_ev@mail.ru
}

Поступило в Редакцию 10 декабря 2020 г.

В окончательной редакции 11 декабря 2020 г.

Принято к публикации 11 декабря 2020 г.

Представлены результаты численного решения задачи сверхзвукового обтекания затупленного ребра, установленного на пластине, вдоль которой развивается пограничный слой. Постановка задачи основана на расчетно-экспериментальной работе Тutty с соавторами (2013), в которой рассмотрен ламинарный режим обтекания при числе Маха внешнего потока равного 6.7. Рассмотрено течение в диапазоне чисел Рейнольдса от $5.0 \cdot 10^{3}$ до $2.0 \cdot 10^{4}$. Установлено, что в некотором интервале значений числа Рейнольдса существуют два устойчивых решения задачи, которые отвечают метастабильным состояниям потока с различной конфигурацией вихревой структуры. Построены бифуркационные диаграммы, показывающие положение центра основного подковообразного вихря и длину отрывной области в зависимости от числа Рейнольдса, и оценено критическое значение числа Рейнольдса, при превышении которого возникает второе решение.

Ключевые слова: высокоскоростные течения, вязко-невязкое взаимодействие, подковообразные вихри, численное моделирование, двойственность решения.

DOI: 10.21883/JTF.2021.05.50687.341-20

\section{Введение}

Натекание сверхзвукового потока на препятствие, установленное на обтекаемой поверхности, приводит к существенно трехмерной структуре течения, которая включает протяженную отрывную область, содержащую систему подковообразных вихрей, а также область сложного ударно-волнового взаимодействия [1-15]. Эффекты вязко-невязкого взаимодействия характеризуются сильно неоднородным распределением теплового потока на обтекаемой поверхности, значения которого могут в несколько раз превышать значения в невозмушенном пограничном слое [4-7,9,13-14]. Изучение структуры потока в подобной конфигурации и правильное предсказание характеристик теплообмена важно как с практической точки зрения, в частности, для аэрокосмической отрасли [16], так и в фундаментально-теоретическом плане.

При взаимодействии пограничного слоя с препятствием в виде затупленного ребра структура потока и картина локального теплообмена зависят от большого числа параметров, таких как свойства среды, характеристики набегающего пограничного слоя, геометрия рассматриваемой конфигурации. В последнее время интерес к данной тематике опять возрос с явно выраженным уклоном в сторону использования методов вычислительной гидрогазодинамики [4-8,13-14], позволяющих исследовать структуру потока для широкого диапазона параметров. Исследования проводятся как для ламинарных режимов течения [3-7,12-15], так и для турбулентных, а также переходных режимов [8-11].

В настоящей работе представлены методика и результаты численного моделирования сверхзвукового ламинарного обтекания затупленного ребра, установленного на пластине, вдоль которой развивается пограничный слой. Постановка задачи основана на данных расчетноэкспериментальной работы [4], в которой изучалась структура ламинарного потока перед обтекаемым телом при числе Маха набегающего потока $\mathrm{M}=6.7$ для трех значений числа Рейнольдса, построенного по диаметру затупления: $\operatorname{Re}_{D}=1.25 \cdot 10^{4}, 2.50 \cdot 10^{4}, 3.75 \cdot 10^{4}$. Численные решения, полученные в цитируемой работе, трактовались авторами как стационарные и единственно возможные. В более позднем расчетном исследовании [5] было показано, что уже при $\operatorname{Re}_{D}=2.50 \cdot 10^{4}$ решение является нестационарным.

Недавно нами было показано [15], что при наименьшем из перечисленных чисел Рейнольдса, $\operatorname{Re}_{D}=1.25 \cdot 10^{4}$, существуют два устойчивых стационарных решения задачи с различной конфигурацией вихрей в отрывной области. По сути, это можно рассматривать, как еще один случай проявления хорошо известного свойства возможной неединственности сверхзвукового обтекания тела, системы тел или их отдельных элементов (см., например, [17-21]). Физическая сторона этого свойства заключается в сильной нелинейности газодинамических процессов. В условиях возможной неединственности реализующаяся картина течения определятся не только геометрическими и физическими параметра- 


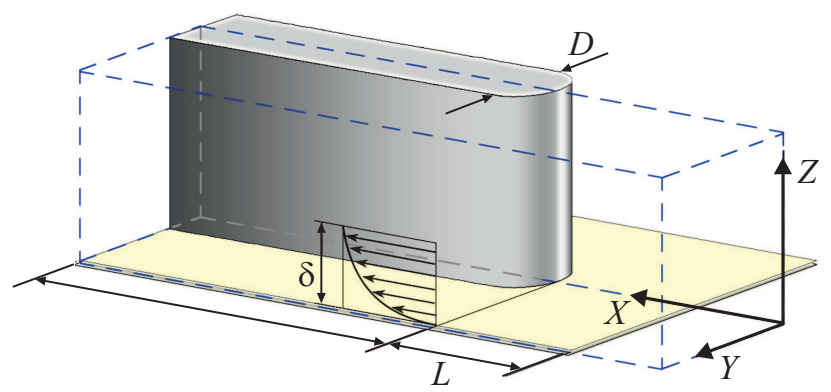

Рис. 1. Расчетная область.

ми, но и предысторией формирования потока; другими словами, проявляется аэродинамический гистерезис.

В настоящей работе, продолжающей исследования, начатые в [15], представляются результаты расчетов, проведенных для интервала изменения числа Рейнольдса от $5.0 \cdot 10^{3}$ до $2.0 \cdot 10^{4}$. Исследуется двойственный характер решения при превышении числом Рейнольдса некоторого критического значения.

\section{1. Постановка задачи}

Расчетная область для рассматриваемой задачи сверхзвукового обтекания совершенным вязким газом затупленного ребра, установленного на пластине, показана на рис. 1. Течение предполагается симметричным относительно плоскости $X Z$, соответственно расчеты проводятся для половины рассматриваемой конфигурации.

Моделируемое течение определяется следующим набором безразмерных параметров: числом Маха набегающего потока $\mathrm{M}$, числом Рейнольдса $\mathrm{Re}_{D}$, числом Прандтля $\mathrm{Pr}$, температурным фактором $T_{\mathrm{w}} / T_{\infty}$, относительной длиной пластины $L / D$ и показателем адиабаты $\gamma$. Следуя [4], течение рассчитывалось при следующих фиксированных параметрах: $\mathrm{M}=6.7, T_{\mathrm{w}} / T_{\text {in }}=4.75, \operatorname{Pr}=0.7$, $\gamma=1.4, L=145 \mathrm{~mm}$ - протяженность пластины до места сопряжения. Расчеты проведены при значениях числа Рейнольдса $\operatorname{Re}_{D}$, лежащих в интервале от $5.0 \cdot 10^{3}$ до $2.0 \cdot 10^{4}$; число Рейнольдса варьировалось посредством изменения диаметра передней затупленной кромки $D$ от 1 до $4 \mathrm{~mm}$.

На входной границе расчетной области задается однородный поток, на поверхности тела и пластине условие прилипания. Поверхности тела и пластины находятся при постоянной температуре $T_{\mathrm{w}}$. На боковых и верхних границах задаются неотражающие граничные условия, на выходе - условие нулевого градиента рассчитываемых переменных.

\section{2. Вычислительные аспекты}

Для расчетов использовался конечно-объемный „неструктурированный“ программный код SINF/Flag-S, разрабатываемый в Институте прикладной математики и механики СПбПУ. Решались полные трехмерные уравнения Навье-Стокса для термически и калорически совершенного газа. Зависимость коэффициента вязкости от температуры определялась формулой Сазерленда.

Вычисление конвективных потоков на гранях контрольных объемов проводилось на основе схемы AUSM [22] второго порядка точности. Реконструкция переменных на гранях контрольных объемов неструктурированной сетки осуществлялась по методу, изложенному в [23,24]. Для обеспечения монотонности решения использовался TVD-подход [25]. Более детальное описание численного метода приведено в [12].

Использовалась сетка, содержащая 10 миллионов ячеек: как показано в [15], сетка данной размерности позволяет обеспечить разрешение ключевых особенностей течения. Все расчеты проводились в нестационарной постановке. Для интегрирования по времени использовался метод „Двойных шагов“ с трехслойной схемой аппроксимации производной по времени (,разностью назад“) второго порядка точности. Безразмерный шаг по времени задавался равным $\Delta t U_{\infty} / L=3.67 \times 10^{-4}$, что обеспечивало значения числа Куранта порядка единицы в большей части расчетной области.

\section{3. Результаты расчетов и их обсуждение}

\section{1. Двойственность решения при $\operatorname{Re}_{D}=1.25 \cdot 10^{4}$}

В работе [15] было показано, что для рассматриваемой конфигурации при $\operatorname{Re}_{D}=1.25 \cdot 10^{4}(D=2.5 \mathrm{~mm})$ существуют два устойчивых стационарных решения с различной конфигурацией вихревой структуры в отрывной области.

Структура течения, отвечающая одному из решений (полученному ранее в $[4,15]$ и ниже обозначаемому как Solution I) приведена на рис. 2, где показаны линии тока и распределение относительного теплового потока

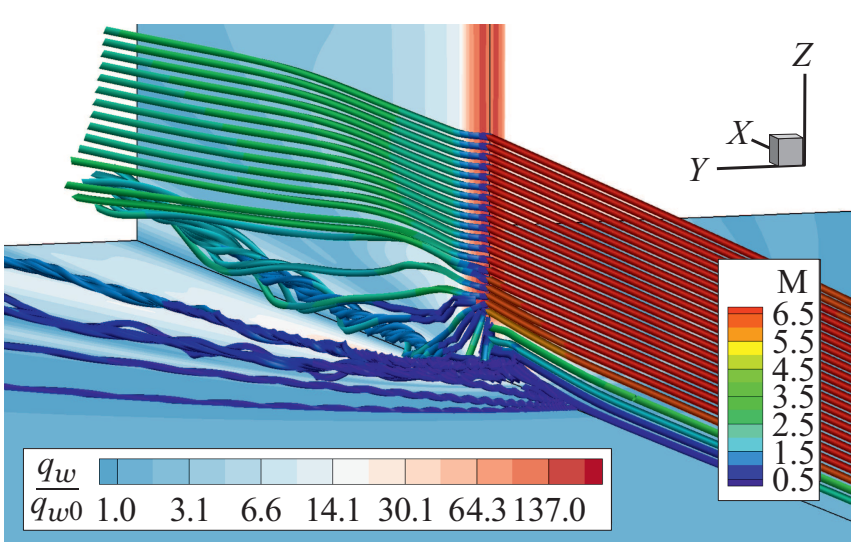

Рис. 2. Иллюстрация трехмерной структуры течения, $\operatorname{Re}_{D}=1.25 \cdot 10^{4}$. 

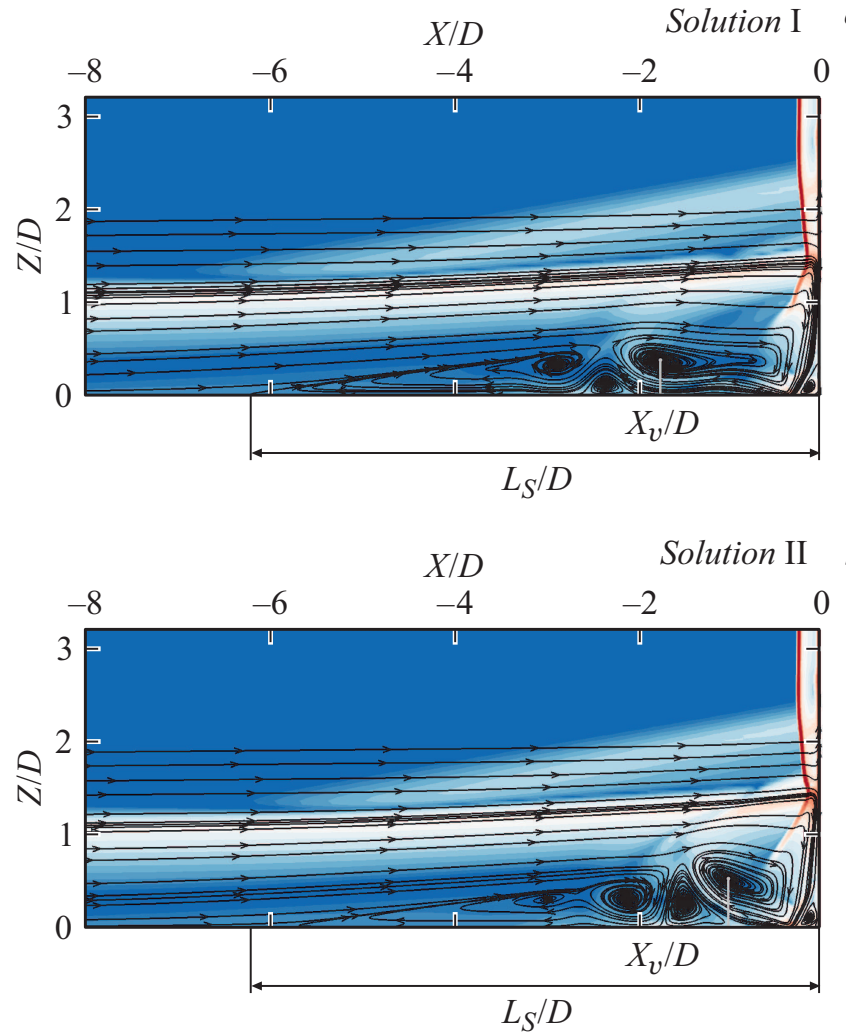

Рис. 3. Структура течения в плоскости симметрии при $\operatorname{Re}_{D}=1.25 \cdot 10^{4}$ : Solution I $(a)$ и Solution II $(b)$.

на поверхности тела и пластины $\left(q_{w 0}-\right.$ значение теплового потока в пластине без препятствия при $x=L)$. Хорошо видны характерные особенности рассматриваемого течения, которые заключаются в формировании отрывной области с системой подковообразных вихрей, влиянии этой области на ударно-волновую картину, а также наличии области повышенной теплоотдачи.

Двойственность решения для данного числа Рейнольдса иллюстрируется на рис. 3, где показана структура потока в плоскости симметрии для двух различных решений („Solution I“ и „Solution II“). Решения отличаются по нормированной протяженности отрывной области $\left(L_{S} / D\right)$, а также по положению оси основного подковообразного вихря $\left(X_{v} / D\right)$. В первом случае $L_{S} / D=6.50, X_{v} / D \approx 1.8$, а для второго решения $L_{S} / D=6.01, X_{v} / D \approx 1.0$. Рис. 4 показывает существенно различные распределения относительного теплового потока, рассчитанные для двух решений, а также поверхностные линии тока.

Как отмечалось выше, в работе [4] было представлено только одно численное решение (Solution I) и отвечающие ему экспериментальные данные. Сопоставление результатов настоящей работы с приведенными в [4] дается на рис. 5.

На рис. 5, $а$ показаны распределения относительного теплового потока вдоль линии симметрии на пластине $\left(q_{w p}\right.$ - значения теплового потока на гладкой пластине без препятствия в соответствующих точках). Видно, что полученное решение, содержащее один глобальный и два локальных максимума, хорошо согласуется с приведенными в работе [4] данными. Рис. 5, $b$ показывает рассчитанную структуру потока в плоскости симметрии, здесь также можно видеть хорошую согласованность результатов.

\section{2. Результаты параметрических расчетов}

Для более полного исследования вопросов двойственности численного решения для рассматриваемой задачи проводились расчеты с „продолжением по параметру“. Фактически в ходе расчетов изменялся диаметр передней кромки ребра $D$; длина пластины и параметры набегающего потока оставались неизменными. Следует отметить, что при этом изменяются значения двух определяющих параметров задачи - числа Рейнольдса $\left(\operatorname{Re}_{D}\right)$ и относительной длины пластины $(L / D)$, соответственно меняется и относительная толщина набегающего пограничного слоя $(\delta / D)$.
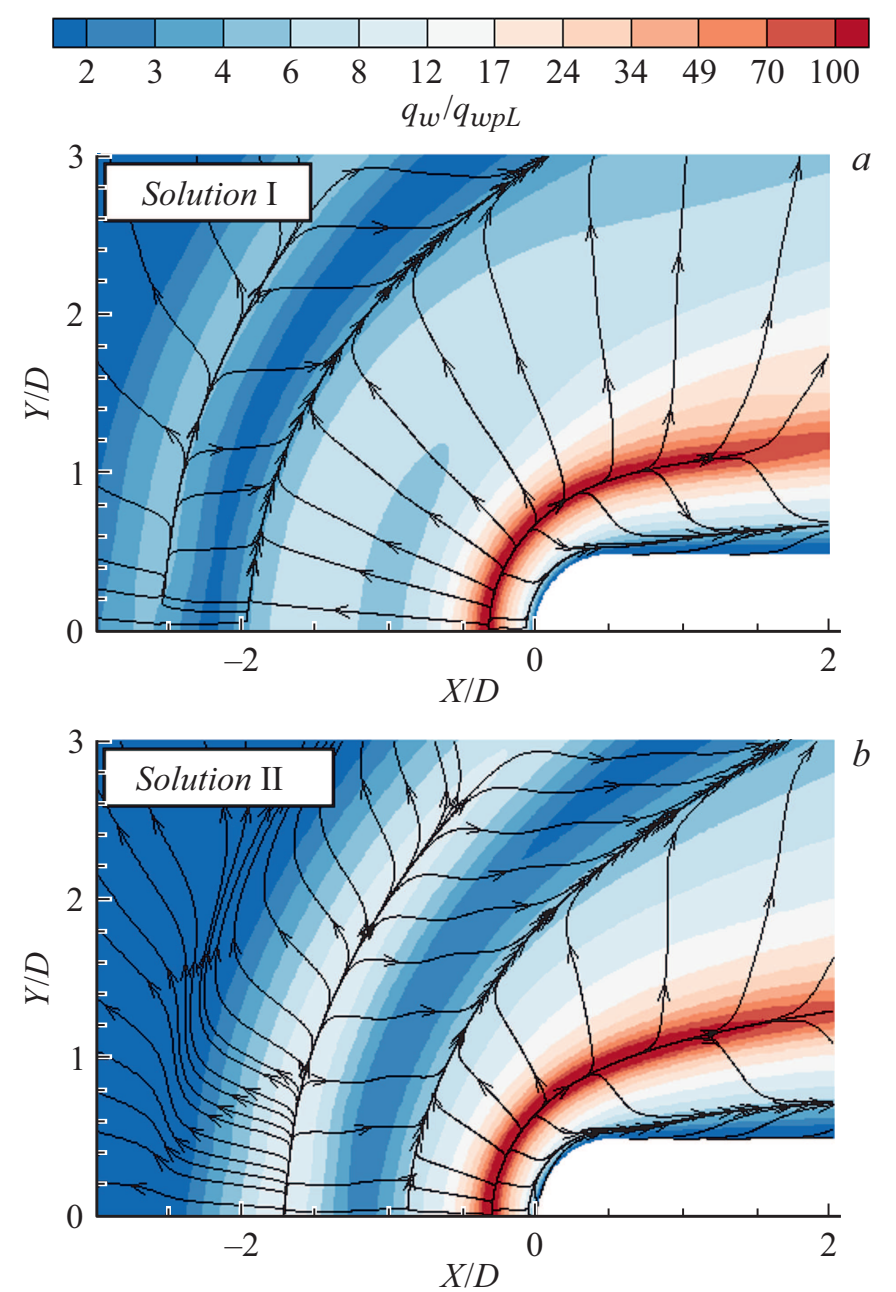

Рис. 4. Распределения числа Стэнтона и поверхностные линии тока на пластине при $\operatorname{Re}_{D}=1.25 \cdot 10^{4}$ : Solution I $(a)$ и Solution II $(b)$. 

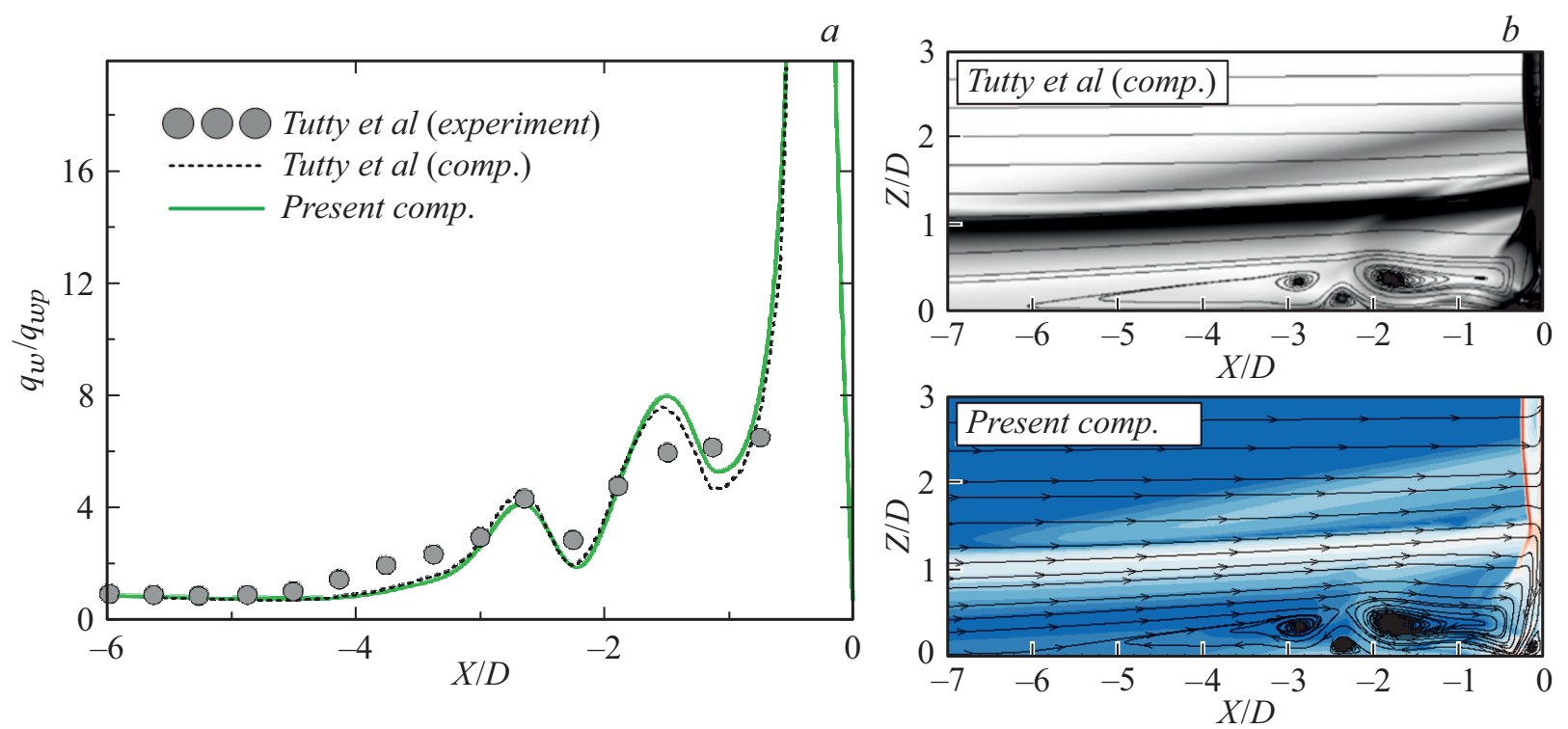

Рис. 5. Сопоставление результатов расчета с данными [4] при $\operatorname{Re}_{D}=1.25 \cdot 10^{4}$ : распределение относительного теплового потока $(a)$ и структура течения в плоскости симметрии $(b)$.
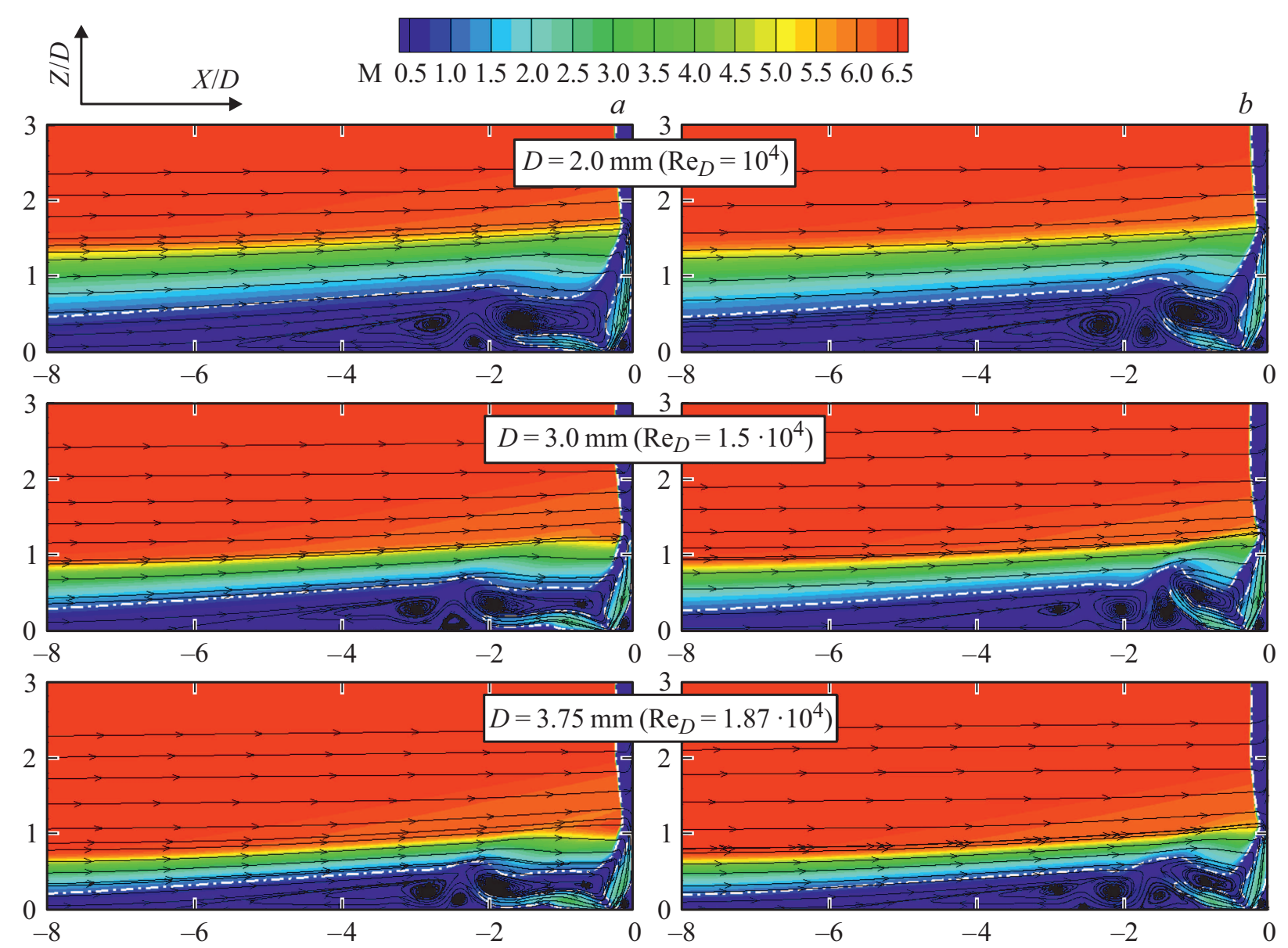

Рис. 6. Поля числа Маха и линии тока в плоскости симметрии (штрихпунктирной линией обозначена звуковая линия) для различных значений диаметра $D$ : Solution I $(a)$ и Solution II $(b)$. 
Рассчитанные параметры отрывной зоны для двух решений

\begin{tabular}{|c|c|c|c|c|c|c|c|}
\hline \multicolumn{4}{|c|}{ Параметры постановки } & \multicolumn{2}{|c|}{ Solution I } & \multicolumn{2}{|c|}{ Solution II } \\
\hline$D, \mathrm{~mm}$ & $\operatorname{Re}_{D}$ & $L / D$ & $\delta / D$ & $X_{v} / D$ & $L_{S} / D$ & $X_{v} / D$ & $L_{S} / D$ \\
\hline 1.00 & $5.0 \cdot 10^{3}$ & 145.00 & 3.00 & 1.130 & 5.53 & & \\
\hline 1.25 & $6.25 \cdot 10^{3}$ & 116.00 & 2.40 & 1.170 & 5.74 & & \\
\hline 1.50 & $7.5 \cdot 10^{3}$ & 96.67 & 2.00 & 1.233 & 5.99 & & \\
\hline 1.75 & $8.75 \cdot 10^{3}$ & 82.86 & 1.71 & 1.316 & 6.17 & & \\
\hline 1.80 & $9.0 \cdot 10^{3}$ & 80.56 & 1.67 & 1.351 & 6.20 & & \\
\hline 1.85 & $9.25 \cdot 10^{3}$ & 78.38 & 1.62 & 1.389 & 6.24 & 1.239 & 6.17 \\
\hline 1.90 & $9.5 \cdot 10^{3}$ & 76.32 & 1.58 & 1.451 & 6.28 & 1.154 & 6.14 \\
\hline 2.00 & $1.0 \cdot 10^{4}$ & 72.50 & 1.50 & 1.540 & 6.34 & 1.096 & 6.12 \\
\hline 2.25 & $1.125 \cdot 10^{4}$ & 64.44 & 1.33 & 1.682 & 6.44 & 1.038 & 6.07 \\
\hline 2.50 & $1.25 \cdot 10^{4}$ & 58.00 & 1.20 & 1.762 & 6.50 & 0.993 & 6.01 \\
\hline 2.65 & $1.325 \cdot 10^{4}$ & 54.72 & 1.13 & 1.832 & 6.55 & 0.982 & 5.99 \\
\hline 2.80 & $1.40 \cdot 10^{4}$ & 51.79 & 1.07 & 1.870 & 6.58 & 0.963 & 5.97 \\
\hline 3.00 & $1.5 \cdot 10^{4}$ & 48.33 & 1.00 & 1.887 & 6.61 & 0.943 & 5.94 \\
\hline 3.13 & $1.56 \cdot 10^{4}$ & 46.40 & 0.96 & 1.891 & 6.60 & 0.929 & 5.93 \\
\hline 3.25 & $1.625 \cdot 10^{4}$ & 44.62 & 0.92 & 1.886 & 6.59 & 0.911 & 5.91 \\
\hline 3.40 & $1.70 \cdot 10^{4}$ & 42.65 & 0.88 & 1.887 & 6.58 & 0.894 & 5.93 \\
\hline 3.75 & $1.87 \cdot 10^{4}$ & 38.67 & 0.80 & 1.876 & 6.55 & 0.873 & 5.97 \\
\hline 4.00 & $2.0 \cdot 10^{4}$ & 36.25 & 0.75 & 1.854 & 6.51 & 0.870 & 6.00 \\
\hline
\end{tabular}

Для проведения расчетов при каждом заданном (текущем) значении диаметра в качестве начальных полей задавались поля величин, полученные для первого и второго решения для предыдущего значения диаметра. Расчеты проводились, начиная со значения $D=2.5 \mathrm{~mm}$ $\left(\operatorname{Re}_{D}=1.25 \cdot 10^{4}\right)$ в сторону увеличения и уменьшения диаметра.

В случае $D=1.8 \mathrm{~mm}\left(\operatorname{Re}_{D}=9.0 \cdot 10^{3}\right)$ при старте с любых полей численное решение оказывается единственным и стационарным (Solution I). Второе стационарное решение возникает при превышении некоторого критического значения числа Рейнольдса, $\operatorname{Re}_{D}$, сr. При дальнейшем увеличении числа Рейнольдса рассчитываемое течение становится нестационарным. Значения числа Рейнольдса, соответствующее переходу к нестационарному режиму течения, различны для двух решений.

На рис. 6 приведены поля числа Маха в плоскости симметрии для нескольких вариантов расчета (в случае нестационарных режимов течения приводятся осредненные во времени характеристики). Видно, что при увеличении диаметра обтекаемого тела основные характеристики течения, свойственные первому и второму решению, в целом сохраняются. В первом решении отрывная область более протяженная и больше „прижа- та“ к поверхности пластины, основной подковообразный вихрь вытянут вдоль пластины, его центр расположен дальше от обтекаемого тела, и вблизи обтекаемого тела образуется еще один дополнительный вихрь. Во втором решении отрывная область короче, центр основного подковообразного вихря расположен ближе к обтекаемому телу.

Сводная таблица содержит значения представительных характеристик течения - положения центра основного подковообразного вихря $\left(X_{v} / D\right)$ и длины отрывной области $\left(L_{S} / D\right)$, рассчитанных в зависимости от варьируемого диаметра передней кромки ребра, там же для сведения даны и значения числа Рейнольдса $\left(\operatorname{Re}_{D}\right)$, относительной длины пластины $(L / D)$ и относительной толщины набегающего пограничного слоя $(\delta / D)$.

На рис. 7 приведены бифуркационные диаграммы, полученные по результатам параметрических расчетов, показывающие зависимость координаты центра основного подковообразного вихря и длины отрывной области от диаметра ребра (числа Рейнольдса). Полученные диаграммы позволяют заключить, что критическое значение диаметра $D_{c}$ находится между 1.8 и $1.85 \mathrm{~mm}$. Видно также, что первое решение с увеличением $\operatorname{Re}_{D}$ меняется эволюционно, а второе решение, 


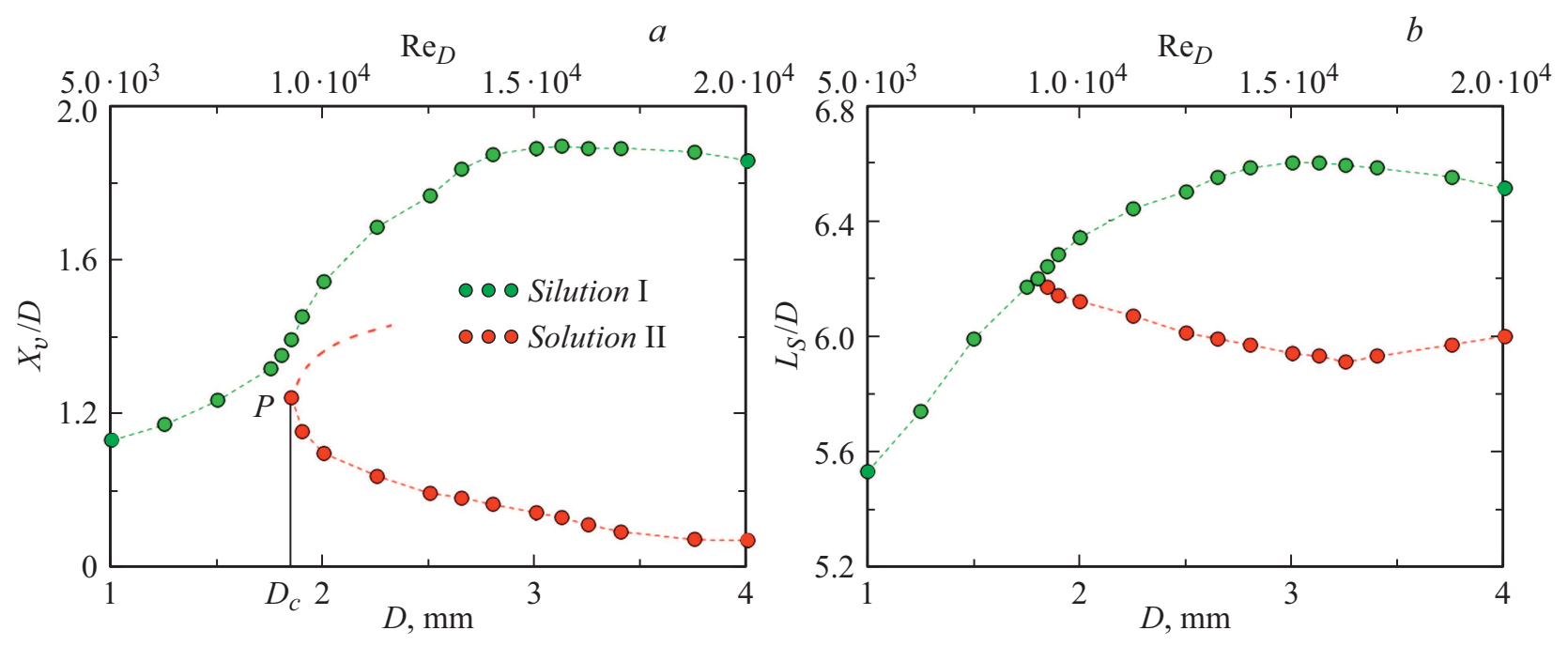

Рис. 7. Бифуркационные диаграммы: положение центра основного подковообразного вихря $(a)$ и длина отрывной области $(b)$.
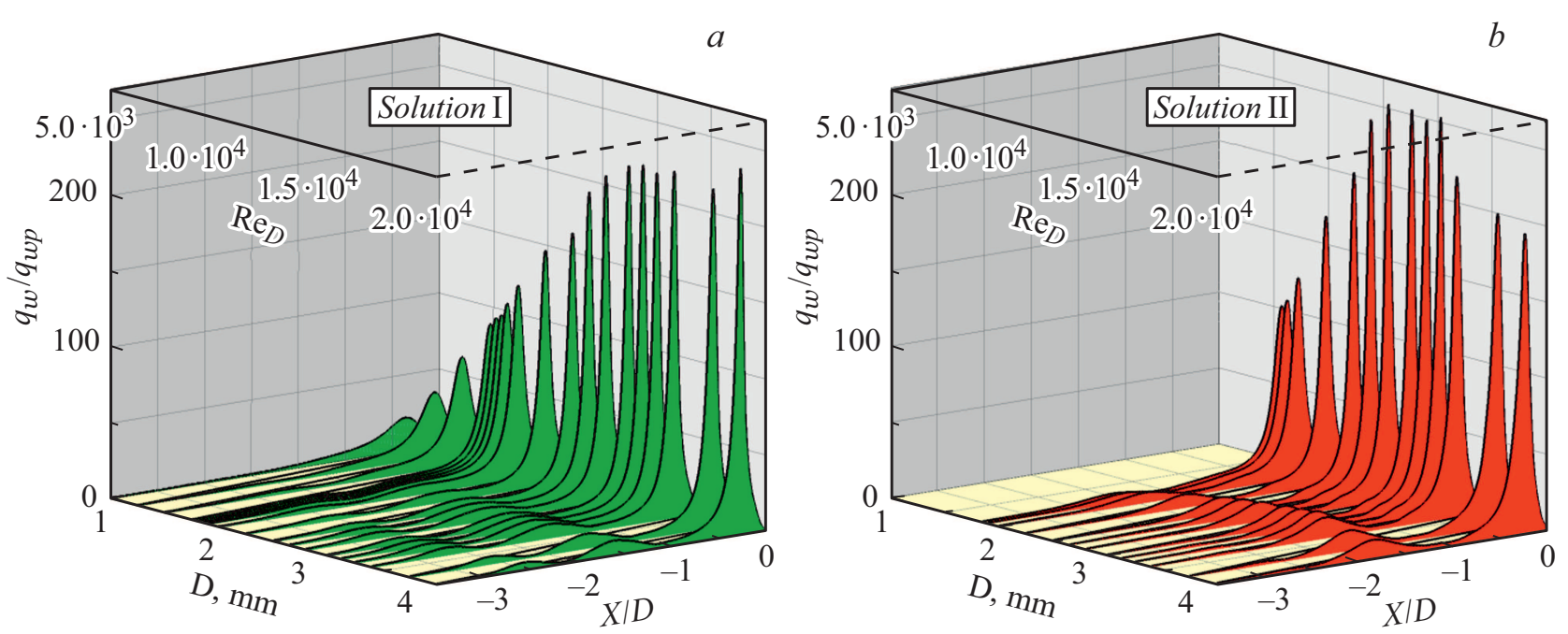

Рис. 8. Распределение теплового потока вдоль линии симметрии для различных значений диаметра D: Solution I (a) и Solution II $(b)$.

по-видимому, является изолированным, существующим при $\operatorname{Re}_{D}>\operatorname{Re}_{D, \text { cr }} \approx 9.25 \cdot 10^{3}$, при этом точка $P$ точка бифуркации (точка поворота), изолированная от ветви решения Solution I.

Длина отрывной области для первого решения с ростом числа Рейнольдса вначале монотонно возрастает, а далее, начиная со значения $\operatorname{Re}_{D} \approx 1.5 \cdot 10^{4}$, постепенно уменьшается. Для второго решения характер зависимости противоположный: уменьшение длины отрывной области наблюдается до значения числа Рейнольдса $\operatorname{Re}_{D} \approx 1.70 \cdot 10^{4}$, начиная с которого идет увеличение длины. Изменения характера зависимости кривых связано с переходом к нестационарным режимам течения.

Следует еще раз подчеркнуть, что представленные бифуркационные диаграммы получены при неизменном положении обтекаемого тела относительно передней кромки пластины $(L=145 \mathrm{~mm})$. Таким образом, относи- тельная толщина набегающего пограничного слоя $\delta / D$, которая является определяющим параметром задачи, меняется при изменении $D$. В принципе, зависимость критического числа Рейнольдса от $\delta / D$ можно определить посредством аналогичных расчетов при варьировании длины пластины $L$ (что является отдельной, весьма ресурсоемкой, задачей).

\section{3. Характеристики теплообмена}

Картина локального теплообмена на поверхности пластины определяется в основном конфигурацией вихрей в отрывной области, и для двух ветвей решения эта картина может значительно отличаться. Для всех рассчитанных вариантов на рис. 8 приведены распределения теплового потока вдоль линии симметрии, представленные в виде $3 D$-диаграммы, где в третьем направлении 


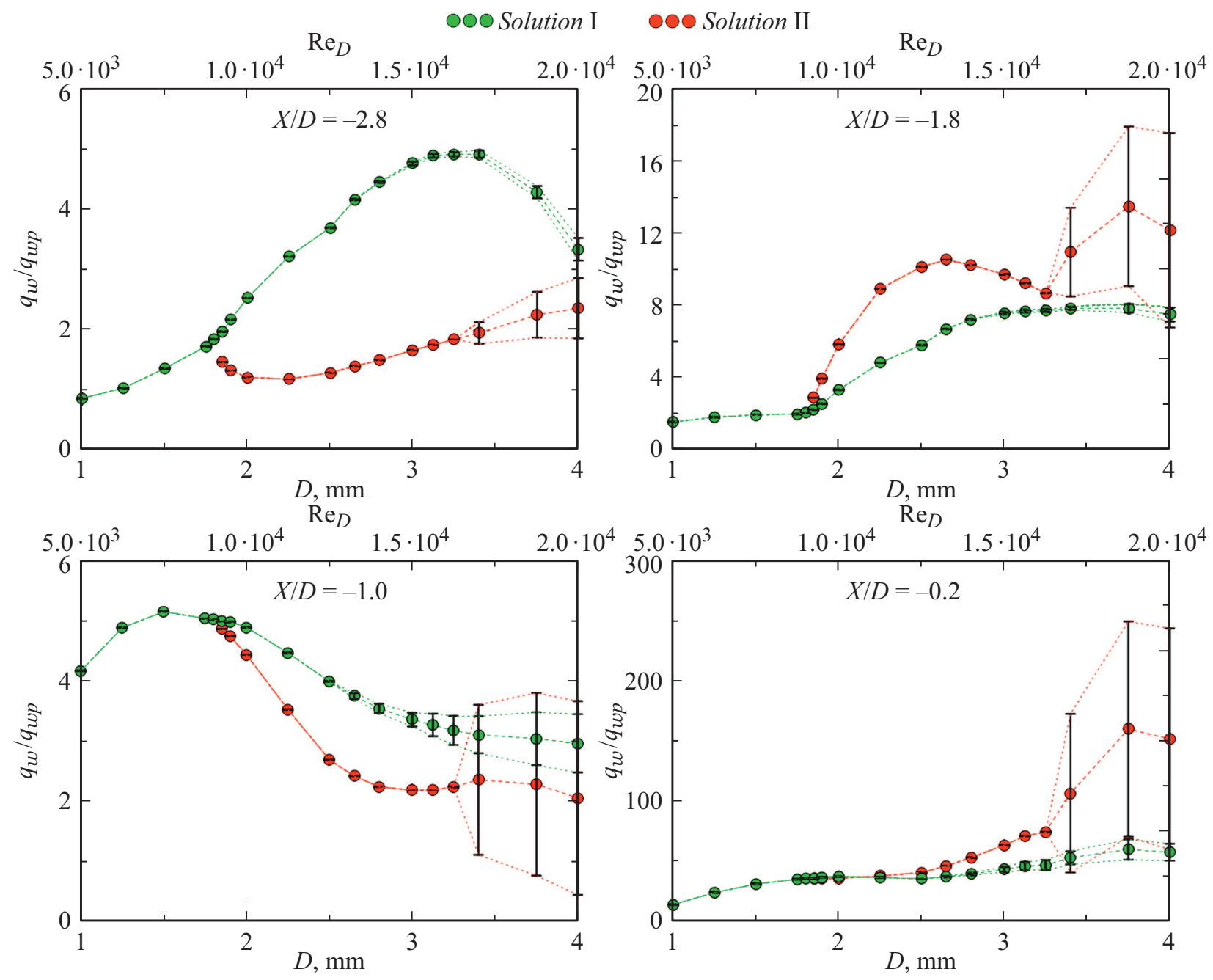

Рис. 9. Значения теплового потока в отдельных точках на пластине вдоль линии симметрии.

отложено значение варьируемого диаметра. Видно, что в случае малого диаметра решение содержит один локальный максимум теплового потока, при увеличении диаметра вихревая структура перед обтекаемым телом усложняется и соответственно меняется распределение теплового потока. Для первого решения характерно наличие двух локальных максимумов на удалении от тела (помимо выраженного главного максимума, расположенного у тела), для второго изолированного решения наблюдается один локальный максимум.

По результатам расчетов анализировалось также поведение значений теплового потока и интенсивности его пульсаций (в случае нестационарных режимов) в отдельных точках на пластине (рис. 9). Вертикальными линиями обозначены среднеквадратичные отклонения для нестационарных режимов.

Из рис. 9 видно, что переход к нестационарному режиму течения для двух решений происходит при разных значениях числа Рейнольдса: для первого - при значении $1.325 \cdot 10^{4}(D=2.65 \mathrm{~mm})$, а для второго при большем значении, равном $1.70 \cdot 10^{4}(D=3.4 \mathrm{~mm})$.
Первому решению отвечает отрывная область большей протяженности, и видимо, поэтому именно оно первым приобретает нестационарный характер.

На приведенных графиках отчетливо видны и различия в общем характере перехода к нестационарному режиму: в случае первого решения интенсивности пульсаций увеличивается постепенно, начиная с малых значений, тогда как для второго решения возникающие пульсации сразу же имеют значительную интенсивность.

\section{Заключение}

Проведены многовариантные параметрические расчеты для трехмерной задачи о взаимодействии сверхзвукового течения вязкого газа с затупленным ребром, установленным на пластине, вдоль которой развивается ламинарный пограничный слой. Расчеты проведены для условий экспериментов [4] при фиксированном расположении тела относительно передней кромки пластины.

Охвачен диапазон изменений числа Рейнольдса от $5.0 \cdot 10^{3}$ до $2.0 \cdot 10^{4}$, включающий стационарные 
и нестационарные режимы течения. Для значения $\operatorname{Re}_{D}=1.25 \cdot 10^{4}$ проведены тестовые расчеты, показавшие хорошее согласие с экспериментальными и расчетными данными [4] для одного из двух возможных стационарных решений задачи.

Исследованы вопросы двойственности решения в рассматриваемом диапазоне числа Рейнольдса. Построены бифуркационые диаграммы, на основе которых оценено критическое значение числа Рейнольдса $\left(\operatorname{Re}_{D, \text { cr }} \approx 9.25 \cdot 10^{3}\right)$, при превышении которого возникает второе (изолированное) решение. Проанализированы основные особенности двух решений, эволюционирующих с ростом числа Рейнольдса, и оценены значения числа Рейнольдса, при которых происходит переход к нестационарным режимам течения.

Исследованы изменения в локальной теплоотдаче, обусловленные ростом числа Рейнольдса. Отмечены характерные особенности распределений теплового потока для решений двух типов.

\section{Благодарности}

Вычисления проводились с использованием ресурсов суперкомпьютерного центра Санкт-Петербургского политехнического университета Петра Великого (www.scc.spbstu.ru).

\section{Конфликт интересов}

Авторы заявляют, что у них нет конфликта интересов.

\section{Список литературы}

[1] Д.М. Войтенко, А.И. Зубков, Ю.А. Панов. Известия АН СССР. Механика жидкости и газа, 1, 121 (1966).

[2] В.С. Адуевский, К.И. Медведев. Известия АН СССР. Механика жидкости и газа, 1, 25 (1967).

[3] B. Lakshmanan, S.N. Tiwari. J. Aircraft, 31 (1), 64 (1994).

[4] O.R. Tutty, G.T. Roberts, P.H. Schuricht. J. Fluid Mechan., 737, 19 (2013)

[5] Y.Q. Zhuang, X.Y. Lu. Procedia Eng., 126, 134 (2015).

[6] M. Mortazavi, D. Knight. Shock Wave Laminar Boundary Layer Interaction at a Hypersonic Flow Over a Blunt Fin-Plate Junction. 55th AIAA Aerospace Sciences Meeting (American Institute of Aeronautics and Astronautics, Reston, Virginia, 2017)

[7] M. Mortazavi, D. Knight. AIAA J., 57 (8), 3506 (2019).

[8] S.A. Lindörfer, C.S. Combs, P.A. Kreth, R.B. Bond, J.D. Schmisseur. Shock Waves, 30 (4), 395 (2020).

[9] V. Borovoy, V. Mosharov, V. Radchenko, A. Skuratov. The shock-waves interference in the flow around a cylinder mounted on a blunted plate. 7Th European Conference For Aeronautics And Aerospace Sciences. 2017. P. 1.

[10] N.T. Clemens , V. Narayanaswamy. Annu. Rev. Fluid Mech., 46 (1), 469 (2014).

[11] C.S. Combs, E.L. Lash, P.A. Kreth, J.D. Schmisseur. AIAA J., 56 (4), 1588 (2018).
[12] Е.В. Колесник, Е.М. Смирнов, А.А. Смирновский. Научнотехнические ведомости СПбГПУ. Физико-математические науки, 12 (2), 7 (2019). DOI: 10.18721/JPM.12201

[13] E.V. Kolesnik, A.A. Smirnovsky. J. Phys. Conf. Ser., 1400 (077030), 077030 (2019).

[14] Е.В. Колесник, Е.М. Смирнов. ЖТФ, 90 (2), 185 (2020).

[15] Е.В. Колесник, А.А. Смирновский, Е.М. Смирнов. Письма в ЖТФ, 46 (12), 10 (2020).

[16] R.H. Korkegi. AIAA J., 9 (5), 771 (1971).

[17] А.И. Гужавин, Я.П. Коробов. Изв. АН СССР. МЖГ, 2, 116 (1984).

[18] И.В. Колин, В.Г. Марков, Т.И. Трифонова, Д.В. Шуховцов. ЖТФ, 74 (2), 124 (2004).

[19] А.Н. Кудрявцев, Д.Б. Эпштейн. Изв. РАН. МЖГ, 3, 122 (2012).

[20] С.В. Гувернюк, А.Ф. Зубков, М.М. Симоненко, А.И. Швец. Изв. РАН. МЖГ, 4, 136 (2014).

[21] Y.-Ch. Hu, W.-F. Zhou, G. Wang, Y.-G. Yang, Zh.-G. Tang. Phys. Fluids, 32 (11), 113601 (2020).

[22] M.S. Liou, C.J. Steffen. J. Comp. Phys., 107 (1), 23 (1993).

[23] C. Le Touze, A. Murrone, H. Guillard. J.Comp. Phys., 284, 389 (2015).

[24] П.А. Бахвалов, Т.К. Козубская. Математическое моделирование, 28 (3), 79 (2016).

[25] A. Harten. J. Comp. Phys., 49 (3), 357 (1983). 